\title{
A Rare Case of Prolactin Secreting Pituitary Carcinoma with Extra-cranial Metastasis
}

Tamar Saeed ${ }^{1}$, Jane Halliday², Christine May ${ }^{1}$, Aparna Pal ${ }^{1}$, Olaf Ansoge ${ }^{12}$, Simon Cudlip ${ }^{2}$, Bahram Jafar-Mohammadi ${ }^{1,2}$

1. Oxford Centre for Diabetes, Endocrinology and Metabolism, Oxford University Hospitals NHS Trust

2. Department of Endocrinology and Neuropathology and Neurosurgery , Oxford University Hospitals NHS Foundation Trust

Introcuction:

\section{Pituitary carcinom}

that fortunately account for $0.1 \%$ of all pituitary tumours. The diagnosis of malignant lesion of the pituitary is ch

structures and the presence of metastasis. Usually the presence of metastasis is intracranial, but cases of extrarani
systemic metastases ). One of the biggest challenges with diagnosis of pituitary carcinoma is that from an endocrin

to benign pituitary tumors as in our case of malignant prolactinoma Such locally invasive and rapidly growing tume standpoint, these tumors often behave identicalily therapy may be required.

\section{Case :}

- A 71-year-old gentleman presented in 2008 with bitemporal hemianopia with pituitary apoplexy compressing the optic chiasm (figure 1).

- Prolactin was $55287 \mathrm{mU} / \mathrm{L}$, with anterior panhypopituitarism

- Due to visual field defact and size of lesion he underwent transphenoidal adenectomy (TSA)

- Histology was in keeping with a lactotroph adenoma with MiB-1 index of 3-5\%, co expression of THS $10 \%$ with no clinical significance and no overexpression of p53

- Post TSA Prolactin remained elevated at $35633 \mathrm{mU} / \mathrm{L}$ and dopamine agonist therapy was commenced with initial good biochemical response.

- Visual fields recovered and Prolactin was normal until 2013.

- He re-presented in 2014 with new visual field defect and Prolactin of $32558 \mathrm{mU} / \mathrm{L}$, escalation of $\mathrm{C}$ abergoline therapy was intended with poor results.

- He underwent debulking surgery in February 2015 due to progression and optic chiasm compression (figure 2).

- Hystopathological features showed atypical pituitary adenoma (mammotroph) with very highmitotic rate and MIB-1 index 20 $30 \%$ with moderate to strong nuclear staining for $\mathrm{p} 53$ in contrast to the previous sample from 2008 .Although the presence of brisk mitotic activity suggests a higher likelihood of invasion and perhaps the eventual occurrence of metastatic spread, it is not an indicator of malignancy per se.

- The post operative MRI scan in December 2015 confirmed rapid regrowth of the tumour (figure 3 ) and Radiotherapy were performed.

- His Prolactin remained stable until September 2017. Slow rise in Prolactin was seen initially with no change in pituitary MRI findings.

- Between March and May 2018 the rate of rise of the Prolactin increased and reached $65807 \mathrm{mU} / \mathrm{L}$. MRI in May 2018 demonstrated two large extra-axial intra-dural enhancing masses at the level of C1-C2 junction with risk of neuronal compression (figure 4).
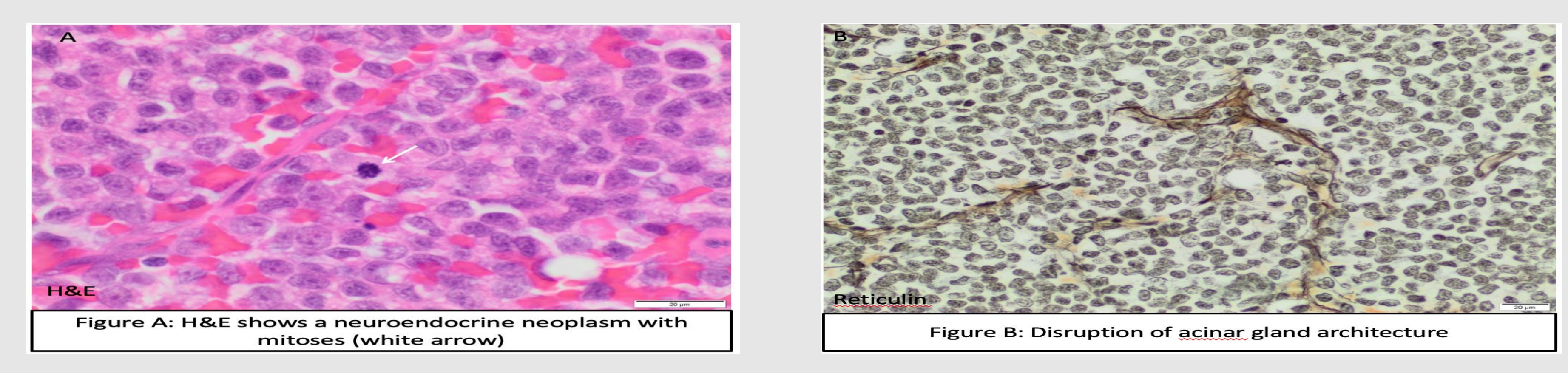

:

\section{(a)}

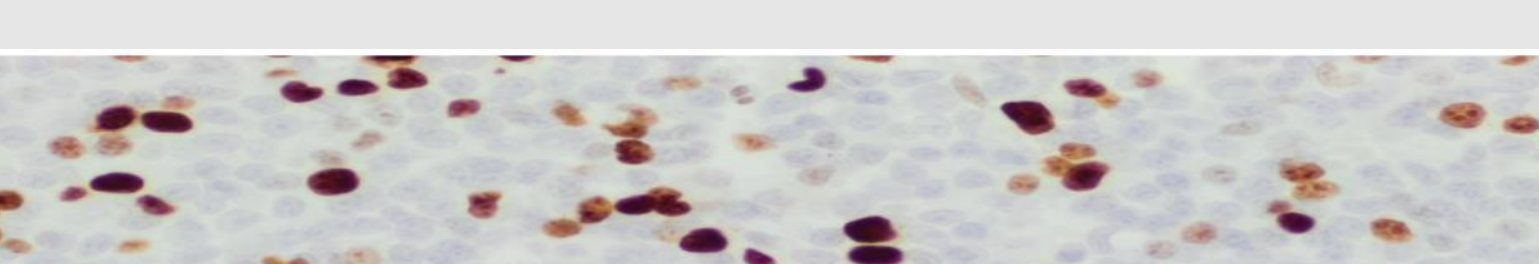

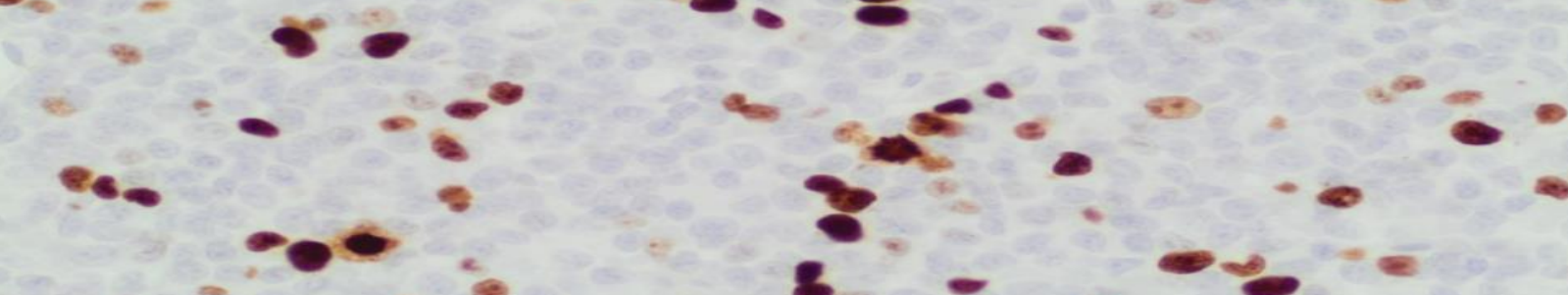

Figure D. Verv hiligh mitotic index of approximately $20.30 \%$

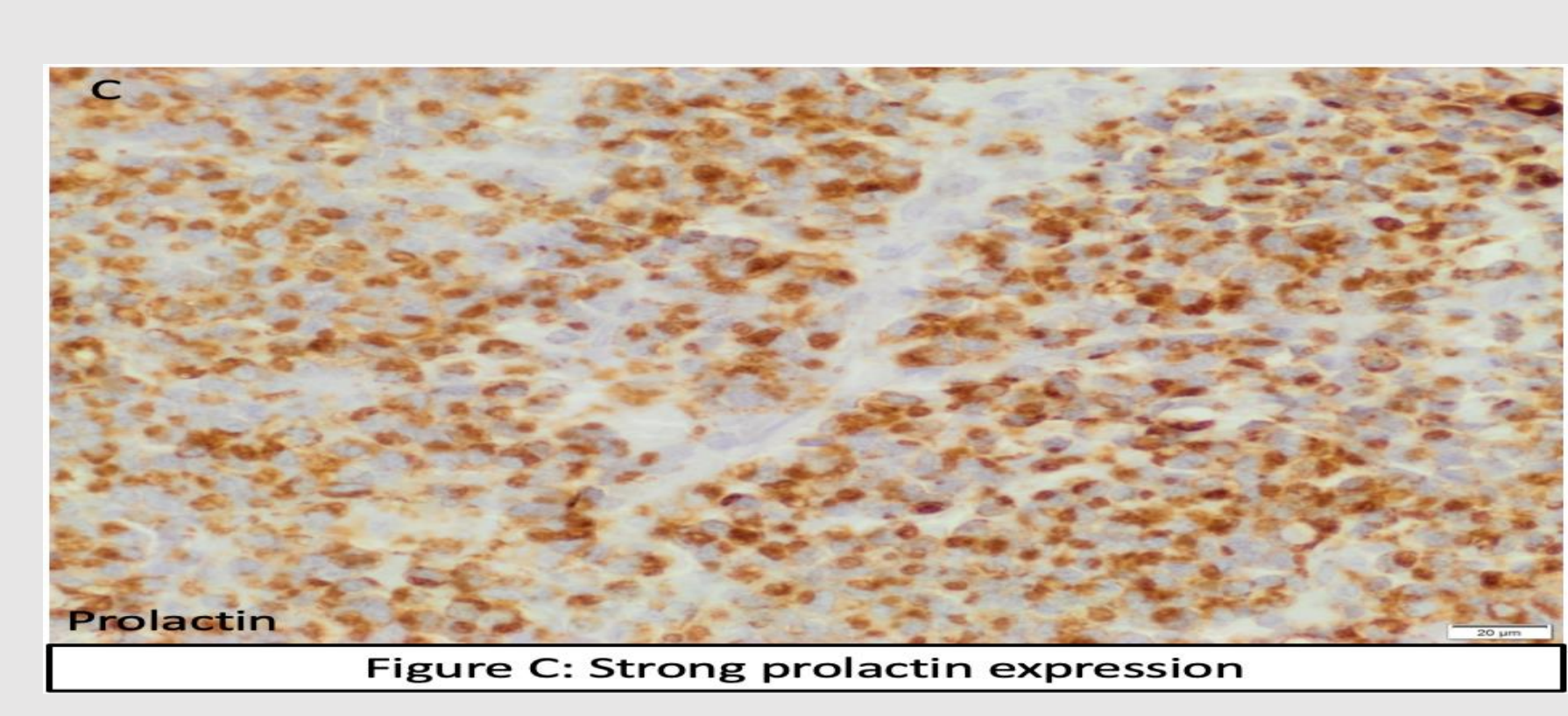

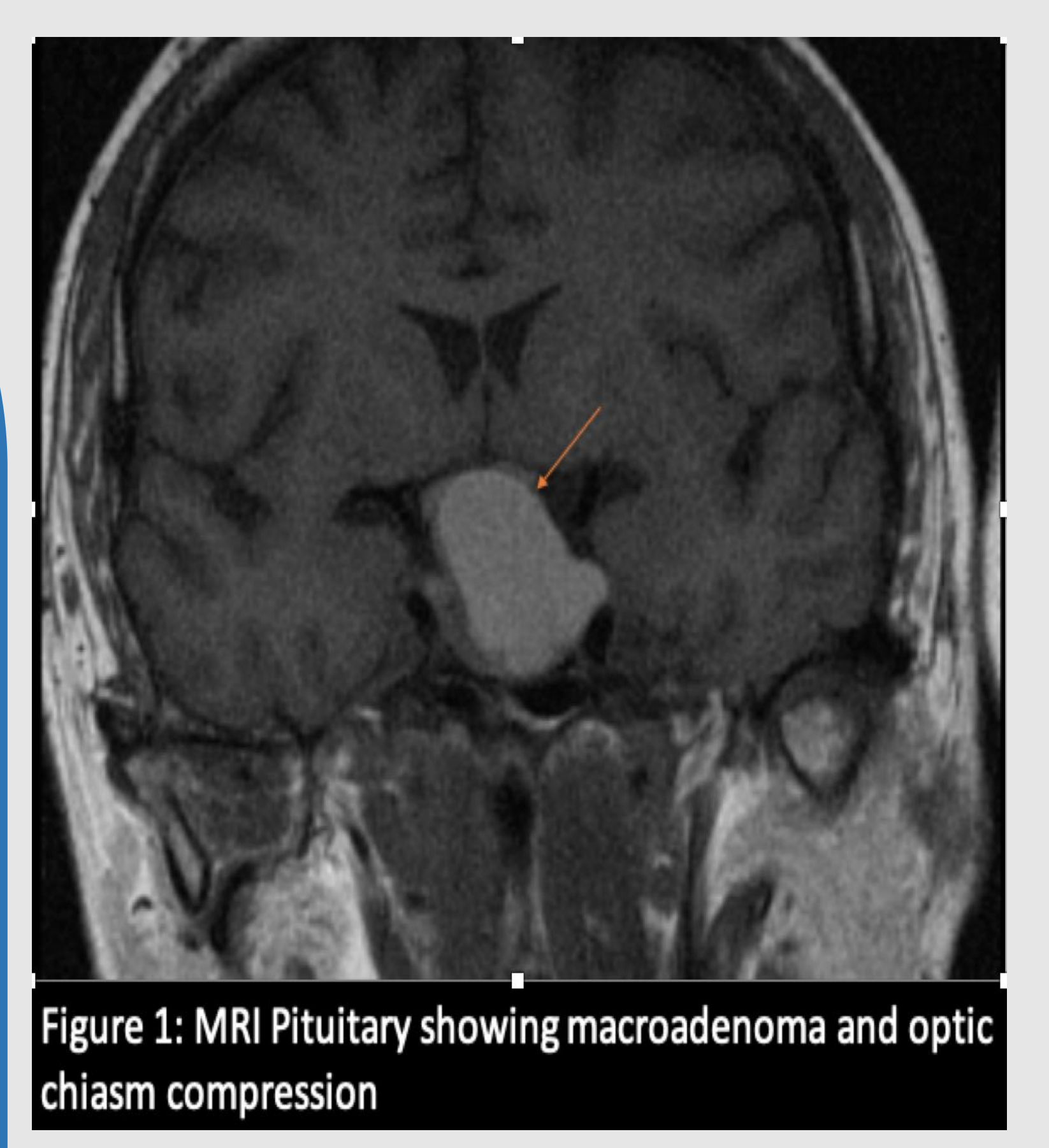
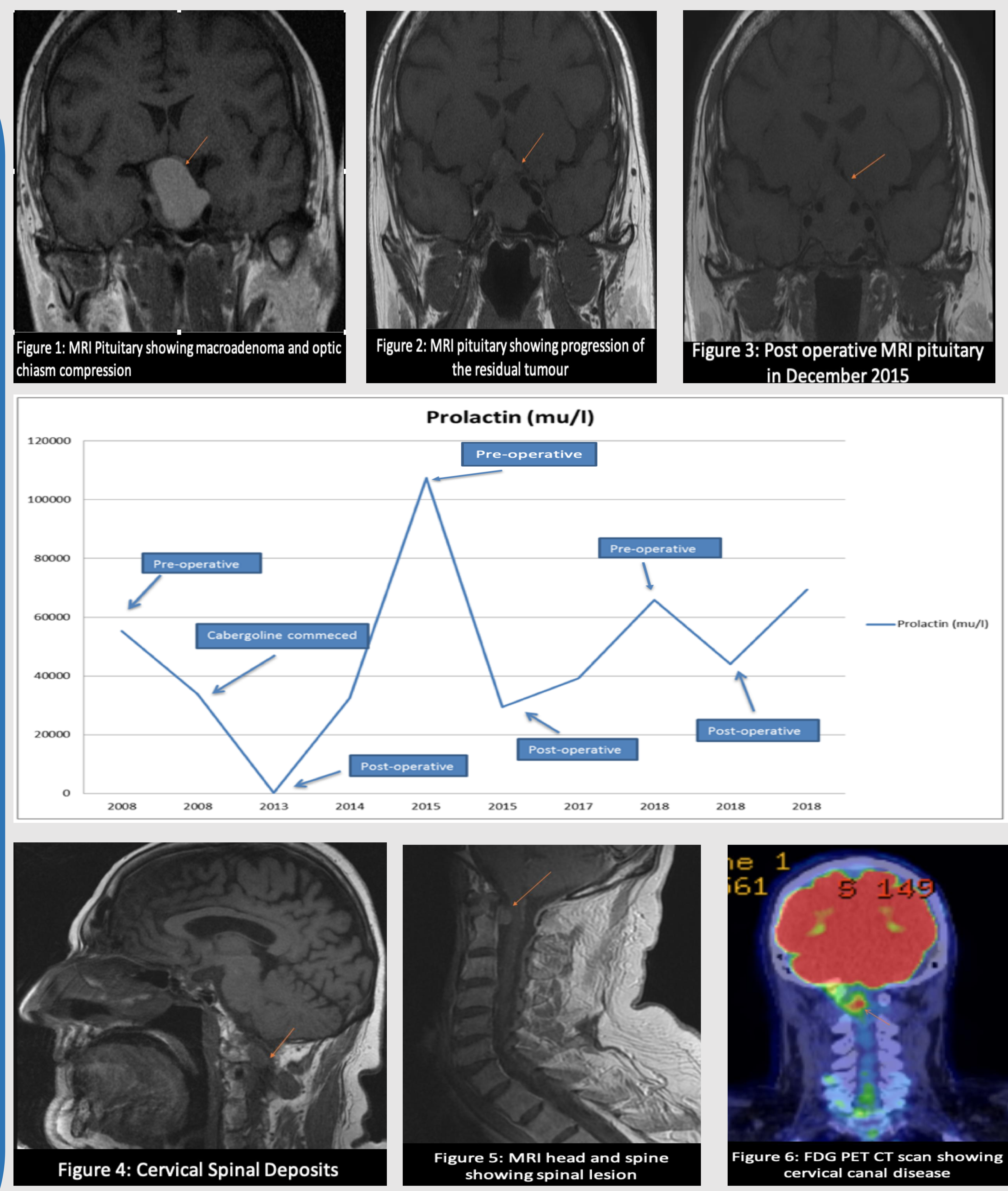

Treatment:

- The patient underwent resection of the pituitary lesion (figures $A, B, C$ and D) and right intradural extramedullary lesion.

- Histology was consistent with a metastatic deposit with raised MiB-1(20-30\%), confirming lactotroph carcinoma.

- Post operatively the prolactin level to $50460 \mathrm{MU} / \mathrm{L}$.

- FDG PET CT scan showed moderately FDG avid cervical canal residual disease (figure 6).

- MRI head and whole spine showed multifocal spinal CSF drop metastasis (figure 5).

- Patient was underwent further radiotherapy and has commenced on

Temozolomide therapy with no severe adverse effects and is awaiting follow up MRI. 